\title{
Estudio de algunos hallazgos numismáticos encontrados en Segovia
}

\author{
manuel abad Varela *
}

Aunque contamos con algunas publicaciones dedicadas a las acuñaciones de Segovia, baste recordar, por ejemplo, la obra de Somorrostro ${ }^{1}$ y fundamentalmente la de Castro $\mathrm{M}^{2}{ }^{a}$ del Rivero ${ }^{2}$, sin embargo, hasta época relativamente reciente, no se tenían apenas referencias que nos mostrasen los hallazgos numismáticos encontrados en la provincia de Segovia, como, por otro lado hubiese sido lo habitual en una provincia con vestigios romanos tan importantes. Esto no quiere decir que no se hubiesen producido sino que, sencillamente, no se llegaron a publicar.

Tenemos entonces que, hasta nuestra última década, solamente se conocian los hallazgos de Castiltierra (Fresno de Cantespino) por el trabajo de Emilio Camps ${ }^{3} \mathrm{y}$, principalmente, los de Duratón por la infatigable labor de Antonio Molinero Pérez ${ }^{4}$.

* UNED. 1820

1 Gomez de Somorrostro, A., El acueducto y otras antigüedades de Segovia, Madrid

2 DEL Rivero, C. M.a. Segovia Numismática, Segovia 1928.

"Camps y Cazorla, E., "Tejidos visigodos de la Necrópolis de Castiltierra", Anuario del Cuerpo Facultativo de Archiveros, Bibliotecarios y Arqueológos, Homenaje a Mélida, II, 1934, págs. 87-96.

4 Molinero Perez, A., "La necrópolis visigoda de Duratón (Segovia). Materiales de tipo bizantino", IV CNA (Elche 1948), pags. 497-505; La necropolis visigoda de Duratón (Segovia). Excavaciones del Plan Nacional de 1942 y 1943, AAH. IV, 1948; Excavaciones arqueológicas antiguas y modernas en Durarón (Segovia), Instituto Diego de Colmenares, Segovia 1949; “Diez años de Arqueologia Segoviana: labor de la Comisaria de Excavacio- 
Es a partir de 1981 cuando el profesor Sagredo inicia los primeros estudios propiamente numismáticos que, a la larga y junto con otros que se vayan produciendo, contribuirán a desvelarnos la economia monetaria de la provincia ${ }^{5}$. Así tendríamos que añadir a éstos el de Carmen Alfaro ${ }^{6}$, que nos presenta antiguos hallazgos (de 1915 a 1922) entre Sepúlveda y Riaza, y, finalmente, los de Juan Francisco Blanco ${ }^{7}$ que se ciñen a los recogidos en torno a Coca.

Sin embargo, debemos destacar que la base para la realización de todos estos estudios la han constituido principalmente las monedas recogidas en Duratón y, fundamentalmente, en Coca.

A tenor de lo publicado hasta ahora tenemos que todavía nos faltan por conocer los hallazgos numismáticos de importantes zonas de la provincia de Segovia, con lo cual resulta necesario, para alcanzar una mejor perspectiva en este campo, dar a conocer todos aquellos hallazgos que se vayan produciendo en el ámbito de la misma.

Con este pequeño trabajo pretendemos aportar nuestro granito de arena presentando quince hallazgos más de monedas romanas que, aparecidas en obras o zanjas dentro de la ciudad de Segovia ${ }^{8}$, fueron recogidas por un aficionado a lo largo de muchos años ${ }^{9}$. Éstas habría que

nes", Estudios Segovianos II, 1950, págs. 639-652; "De la Segovia arqueológica", Segovia, 24-abril-1954; Aportaciones de las excavaciones y hallazgos casuales (1941-1959) al Museo Arqueológico de Segovia, EAE, 72, Madrid 1971; Un hecho digno de ser recordado en su primer cincuentenario: el del descubrimiento de la necrópolis visigoda de Duratón (1929. 1979) (Separata corregida de los artículos publicados bajo el mismo título en El Adelantado de Segovia de los dias 17, 18, 19, 20 y 24 de abril de 1979). Segovia 1979. El trabajo de LaINEZ, M., "Apuntes históricos de Segovia", ES XVI, 1964, pág. 5-432, añade poca información numismática.

"Sagredo San Eustaquio, L.: "Posible tesorillo del siglo Ill», Numisma XXXI, núm. 169-173, 1981, págs. 73-88; "Tesorillo tardorromano de la Meseta Norte", Nvmmvs, 2." serie VIIIVIII, 1984-1985, págs. 47-54; Sagredo San Eustaquio, L. y ArRibas Clemente, E., Circulación y evolución monetaria en la provincia de Segovia en la Antigüedad, Segovia 1987; y Sagredo San Eustaquio, L. y Zumel Menocal, L., "Estudio de monedas tardorromanas procedentes de Coca", Numisma XXXV-XXXVI, núm. 192-203, 1985-86, págs. 9-32.

${ }^{6}$ Alfaro Asins, C.: "Monedas con indicación de procedencia recientemente intregradas en la sección de numismática del M.A.N., I", BMAN III, pág. 146-147. Estas fueron recogidas en prospecciones arqueológicas ordenadas por el Marqués de Cerralbo entre la zona de Sepúlveda y Riaza.

Blanco y Garcia, J. F.: Coca arqueológica, Madrid, 1986; y Moneda y circulación monetaria en Coca (siglos // a.C. y V d.C.), Segovia 1987.

${ }^{8}$ Se nos ha dicho que proceden todas del casco antiguo de la ciudad de Segovia y que fueron encontradas de forma individualizada, pero no se guarda memoria de los lugares exactos en que se hallaron.

${ }^{9}$ Doy las gracias a la familia de D. José García Cabrero por sus atenciones y todas las facilidades ofrecidas para el estudio de las piezas. 
añadirlas a las otras siete encontradas también en Segovia y depositadas en su Museo Arqueológico ${ }^{10}$. Además, damos a conocer, dentro de este conjunto, una, la número dieciséis, encontrada en Espirdo ${ }^{11}$.

\section{CATÁLOGO DE MONEDAS}

\section{Segovia}

N. ${ }^{\circ} 1$

Galba (68)

Ley. anv.: ...MP CAESAR A...

Descripción: Busto laureado a dcha.

Ley. rev.: S C

Descripción: Entre $S$ y $C$, insignia de águila legionaria sobre rayo entre dos estandartes. Debajo del conjunto una linea horizontal.

Ceca: Roma

As; Metal: bronce

Peso: 10,11 gr; Mód.: 28 mm; Gros.: 2,5 mm; Ej.: 6; Cons.: MM con agujero.

Ref. bibliog.: RIC I, pág. 252, $.^{\circ} 18,21$ ó 22; El reverso es similar a: $B M C$ I, pág. $334,{ }^{\circ} 153$, lám. 58, n. $^{\circ} 1$.

\section{N. ${ }^{\circ} 2$}

Domiciano (84-85)

Ley. anv.: IMP CAES DOMITIAN AVG GERM...

Descripción: Busto laureado a dcha.

Ley. rev.: ... / AVGVSTI, en el campo S C

Descripción: Altar

Ceca: Roma

As; Metal: bronce

Peso: 9,85 gr; Mód.: $26 \mathrm{~mm}$; Gros.: $2,5 \mathrm{~mm}$; Ej.: 6; Cons.: M, rota.

Ref. bibliog.: RIC II, pág. 186, n. 251 ó 188, n. 272.

10 Sagredo San Eustaquio, L. Y arribas Clemente, E., Circulación y evolución monetaria..., obra citada, págs. 150-153.

"Agradezco a D. José Antonio Morato Herrero la información y el haberme posibilitado su estudio. 
N. ${ }^{\circ} 3$

Antonino Pio (140-161)

Ley. anv.: ANTONINVS AVG PI VS PP TR P CON...

Descripción: Busto laureado a dcha.

Ley. rev.: S... S ..., en el campo S C

Descripción: Salus en pie a izq. con paludamentum, en su mano dcha. sostiene una pátera con la que alimenta a una serpiente enroscada en un ara y con la izq. un timón sobre globo.

Ceca: Roma

Sex.; Metal: bronce

Peso: $27 \mathrm{gr}$; Mód.: $30 \mathrm{~mm}$; Gros.: $5 \mathrm{~mm}$; Ej.: 12; Cons.: $M$

Ref. bibliog.: RIC III, pág. $112, \mathrm{n}^{\circ} 636$ ó pág. $126, \mathrm{n} .{ }^{\circ} 784$.

$B M C$ IV, pág. 276, n. ${ }^{\circ} 1715$.

N. ${ }^{\circ} 4$

Alejandro Severo (222-¿229?)

Ley. anv.: ...SEV ALE ...

Descripción: Busto laureado a dcha.

Ley. rev.: P... en el campo $S \mathrm{C}$

Descripción: Entre $S$ y $C$ Marte marchando a dcha. sosteniendo lanza con la mano dcha. mientras con la izq. porta trofeo sobre el hombro.

Ceca: Roma

Sex.; Metal: bronce

Peso: 16,38 gr; Mód.: 29 mm; Gros.: $3,5 \mathrm{~mm}$; Ej.: 12; Cons.: MM rota.

Ref. bibliog.: Parecida a las de: RIC IV, pág. 103, n. ${ }^{\circ}$ 390, pág. 105, n. ${ }^{\circ} 424-426$, pág. $107, n .^{\circ} 456-458$ pág. $108, n .^{\circ} 472$; y también a la de: ROBERTSON III, pág. 152, n. ${ }^{\circ} 108$.

N. ${ }^{\circ} 5$

Maximino I (236-238)

Ley. anv.: ...IMINVS PIVS AVG GE...

Descripción: Busto laureado a dcha. con paludamentum y coraza.

Ley. rev.: (tipo pax avgrsti) en el campo S C

Descripción: Paz de pie a izq., sosteniendo una rama con su mano dcha. extendida mientras sostiene con la izq. un cetro que le atraviesa.

Ceca: Roma

Sex.; Metal: bronce

Peso: 7,30 gr; Mód:: $26 \mathrm{~mm}$; Gros.: $3 \mathrm{~mm}$; Ej.: 6; Cons.: MM, rota.

Ref. bibliog.: RIC, IV, $2 .{ }^{a}$ parte, pág. 146, n. ${ }^{\circ} 81$; ROBERTSON, III, pág. $176,{ }^{\circ} 49$ y 52 . 
N. ${ }^{\circ} 6$

Gordiano III (238-239)

Ley, anv.: IMP CAES ... VS AVG

Descripción: Busto laureado a dcha. con paludamentum y coraza.

Ley, rev.: ...L...S AVG, en el campo S C

Descripción: Salus de pie a dcha. con paludamentum, sostiene la pátera con la mano izq. donde va a comer la serpiente que sujeta con la dcha.

Ceca: Roma

Sex.; Metal: bronce

Peso: 11,40 gr; Mód.: 26,5 mm; Gros.: 3 mm; Ej.: 12; Cons.: MM, rota

Ref. bibliog.: RIC IV 3 . $^{\text {a }}$ parte, pág. $44, \mathrm{n} .{ }^{\circ} 260$, a; ROBERTSON, III, pág. 202, n. ${ }^{\circ} 92$.

N. ${ }^{\circ} 7$

Claudio II divinizado (270)

Ley. anv.: ... CLAVD...

Descripción: Busto desnudo con corona radiada a dcha.

Ley. rev.: CONSEC...

Descripción: Altar cuadrado con llama.

Ceca: Roma

Ant.; Metal: aleación

Peso: $1,31 \mathrm{gr}$; Mód.: $15 \mathrm{~mm}$; Gros.: $1,2 \mathrm{~mm}$; Ej.: 12; Cons.: M

Ref. bibliog.: ROBERTSON, IV, pág. $80, \mathrm{n}^{\circ} 8$.

N. ${ }^{\circ} 8$

Probo (279)

Ley. anv.: IMP C PROBVS P F AVG

Descripción: Busto con corona radiada a dcha., paludamentum y coraza.

Ley. rev.: PAX A VGV...।

Descripción: Paz de pie a izq., sosteniendo una rama con su mano dcha. extendida mientras sostiene con la izq. un cetro que le atraviesa.

Exergo: XXIQ Ceca: Siscia, $4 .^{\text {a }}$ oficina.

Ant.; Metal: aleación

Peso: 3,60 gr; Mód: $21 \mathrm{~mm}$; Gros.: 1,2 mm; Ej.: 12; Cons.: M.

Ref. bibliog.: RIC V, 2 parte, ${ }^{\circ}{ }^{\circ} 712$; ROBERTSON IV, pág. 185, n. ${ }^{\circ}$ 218.

N. ${ }^{\circ} 9$

Constantino II (319)

Ley. anv.: IMP CONSTAN TINVS MAX AVG 
Descripción: Busto con casco laureado y coraza a dcha.

Ley. rev.: VICTORIAE LAETAE PRINC PERP

Descripción: Dos victorias de pie, enfrentadas, sostienen un escudo con la leyenda VOT / PR sobre un altar.

Exergo: STR Ceca: Tréveris, $2 .^{a}$ oficina.

Nummus; Metal: bronce

Peso: 2,61 gr; Mód.: 16 mm; Gros.: 1,5 mm; Ej.: 6; Cons.: M, rota. Ref. bibliog.: RIC VII, pág. 182, n. 213.

N. ${ }^{\circ} 10$

Decencio (351-352)

Ley. anv.: D N DECENTIVS NOB CAES

Descripción: Busto desnudo a dcha. con paludamentum y coraza.

Ley. rev.: VICTORIAE DD NN AVG ET CAE

Descripción: Dos victorias de frente una de otra sostienen un escudo con la inscripción VOT / V / MVLT / X.

$\mathrm{SP}$

Exergo: RSLG Ceca: Lugdunum, 2. a oficina.

Maiorina; Metal: bronce

Peso: 3,74 gr: Mód.: $21 \mathrm{~mm}$; Gros.: 1,2 mm; Ej.: 1: Cons.: M.

Ref. bibliog.: RIC VIII, pág. 187, BASTIEN, pág. 178, n. ${ }^{\circ} 177$.

N. ${ }^{\circ} 11$

Constancio II (355-361)

Ley. anv.: ... CONSTAN ... V ...

Descripción: Busto diademado a dcha. con paludamentum y coraza.

Ley. rev.: (tipo fel temp reparatio)

Descripción: Emperador en pie, mirando a izq. con escudo en brazo izq., alanceando a jinete que cae del caballo y levanta un branzo hacia atrás; escudo en el suelo a dcha.

Exergo: ...

Ceca: ...

Ae 3; Metal: bronce

Peso: 1,55 gr.; Mód.: 16 mm; Gros.: 1,2 mm; Ej.: 12; Cons.: MM.

Ref. bibliog.: BRUCK, pág. 20

N. ${ }^{\circ} 12$

Valentiniano I (367-375)

Ley. anv.: D N VALENTINI ANVS ... AVG

Descripción: Busto diademado con roseta a dcha., paludamentum y coraza.

Ley. rev.: SECVRITAS REIPVBLICAE

Descripción: victoria andando a izq. con corona en la mano dcha. y palma en la izq. 
Exergo: ... Ceca: Tréveris, Aquileya, Roma o Tesalónica Ae 3; Metal: bronce

Peso: 2,10 gr; Mód.: $18 \mathrm{~mm}$; Gros.: 1,2 mm; Ej.: 6; Cons.: M, rota.

Ref. bibliog.: BRUCK, págs. 66 y 67.

N. ${ }^{\circ} 13$

Teodosio I (378-383)

Ley. anv.: ...EODO SIV...P...

Descripción: Busto diademado a dcha. con paludamentum y coraza. Ley. rev.: REPARA... REIPV...

Descripción: Emperador de pie, cabeza a la izq., dando su mano dcha. a una mujer torreada y con rodilla en tierra, mientras sostiene en su izq. una victoria coronándole.

Exergo: ASISC Ceca: Siscia, $1 .^{a}$ oficina

Ae 2; Metal: bronce

Peso: $3,90 \mathrm{gr}$; Mód.: $23 \mathrm{~mm}$; Gros.: 1,2 mm; Ej.: 1; Cons.: M.

Ref. bibliog.: $\angle R B C$, pág. 74, n. 1514.

N. ${ }^{\circ} 14$

(378-383)

Ley. anv.:

Descripción: Busto diademado a dcha. con paludamentum y coraza. Ley. rev.: (tipo reparatio reipvb)

Descripción: como la n. ${ }^{\circ} 13$.

Exergo: CONS... Ceca: Constantinopla

Ae 2; Metal: bronce

Peso: 4 gr; Mód.: 22 mm; Gros.: 1,2 mm; Ej.: 12; Cons.: MM.

Ref. bibliog.: $\angle R B C$, pág. $88, \mathrm{n} .{ }^{\circ} 2118-2120$.

N. ${ }^{\circ} 15$

Magno Máximo (383-387)

Ley. anv.: D N MAG MAXI MVS P F AVG

Descripción: Busto con diadema de perlas a dcha., paludamentum y coraza.

Ley. rev.: REPARATIO REIPVB

Descripción: Igual que la $\mathrm{n}^{\circ} 13$.

Exergo: ...CON Ceca: Arlés

Ae 2; Metal: bronce

Peso: 4,30 gr; Mód.: 22 mm; Gros.: 1,5 mm; Ej.: 12; Cons.: R.

Ref. bibliog.: $\angle R B C$, pág. $57, \mathrm{n}^{\circ} 553$. 


\section{Espirdo (Segovia)}

N. ${ }^{\circ} 16$

Claudio II divinizado (270)

Ley. anv.: DIVO CLAVD...

Descripción: Busto desnudo con corona radiada a dcha.

Ley. rev.: CONSECRATIO

Descripción: Altar cuadrado con llama.

Ceca: Roma

Ant.; Metal: aleación

Peso: 1,82 gr; Mód.: 17 mm; Gros.: 1,3 mm; Ej.: 11; Cons.: M

Ref. bibliog.: ROBERTSON, IV, pág. 80, n. $^{\circ} 8$.

\section{ESTUDIO}

Nos hubiese gustado contar con un número más abundante de piezas para realizar un estudio de mayor garantía. Como no es posible, por el momento,analizaremos este conjunto al que sumaremos, cuando ofrezcamos los resultados globales, las otras siete monedas aparecidas en Segovia (ver nota 10 ).

$\mathrm{Si}$ nos atenemos a la representación de los emperadores en las piezas estudiadas ahora, obtenemos el siguiente cuadro:

\begin{tabular}{lc} 
Emperadores & Número \\
\hline Galba & 1 \\
Domiciano & 1 \\
Antonino Pío & 1 \\
Alejandro Severo & 1 \\
Maximino I & 1 \\
Gordiano III & 1 \\
Claudio II, divinizado & 1 \\
Probo & 1 \\
Constantino II & 1 \\
Decencio & 1 \\
Constancio II 1 en Espirdo) \\
Valentiniano I & 1 \\
Teodosio I & 1 \\
Espirdo & 1 \\
Magno Máximo & 1 \\
\end{tabular}



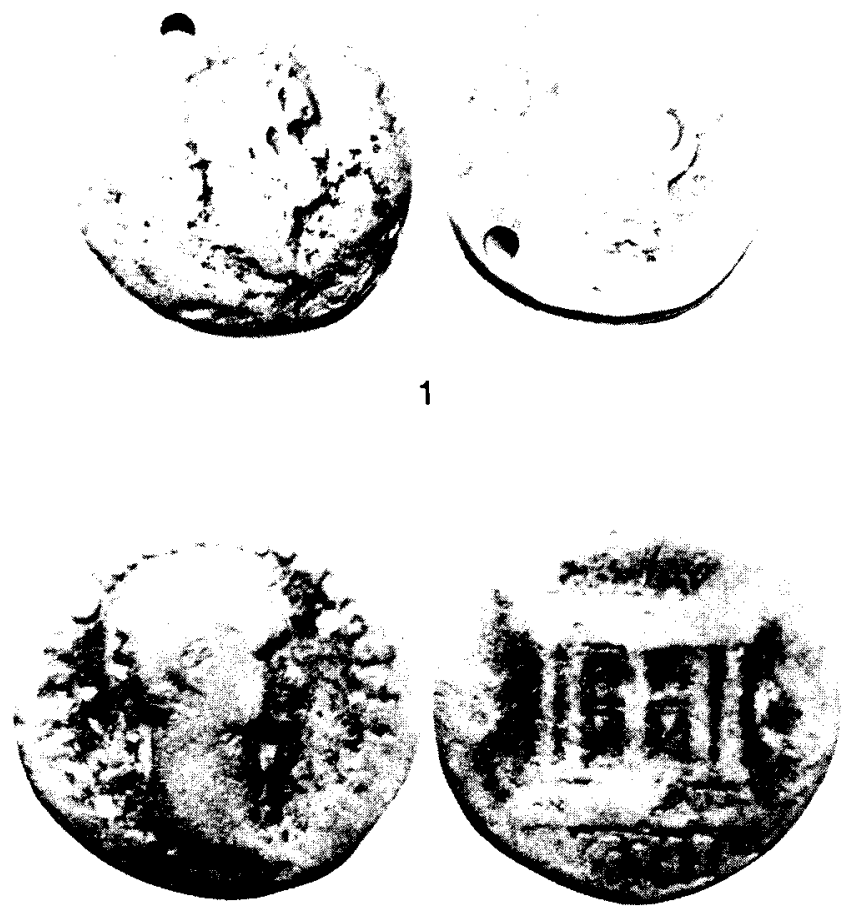

2

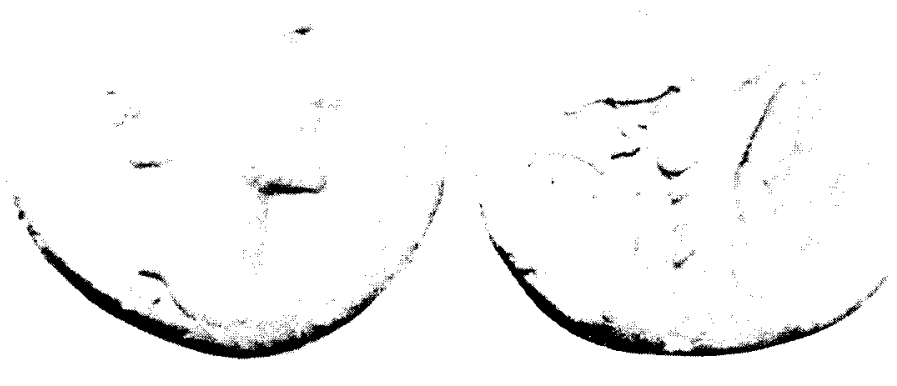



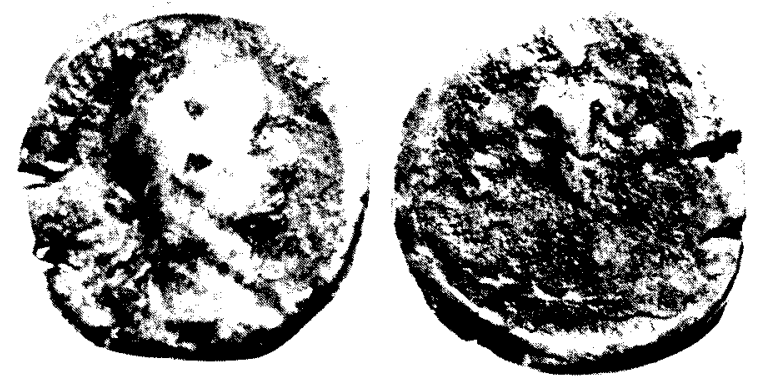

4
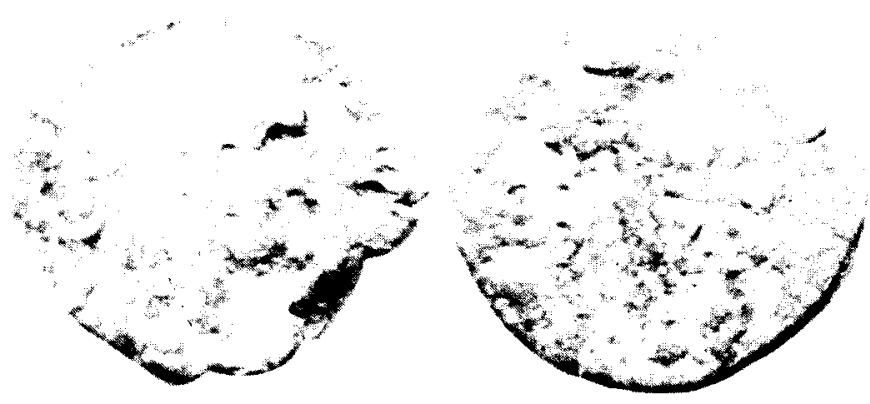

5
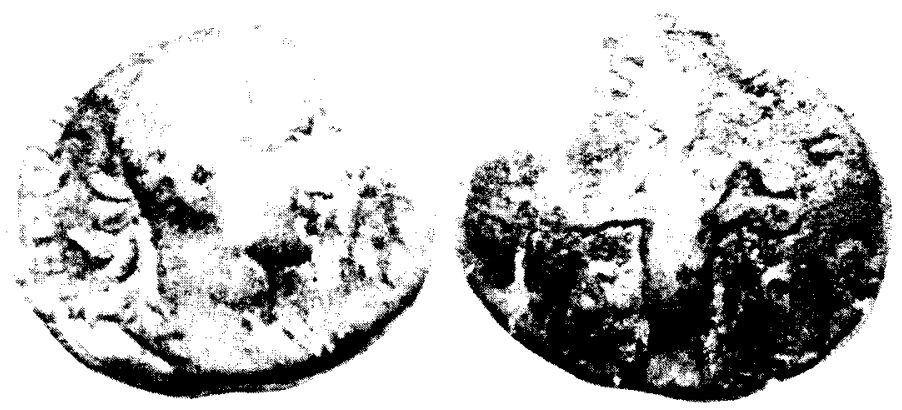

6 

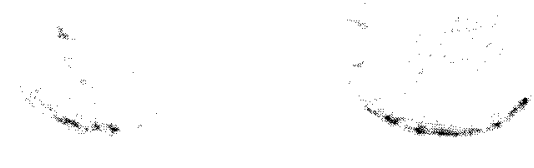

7
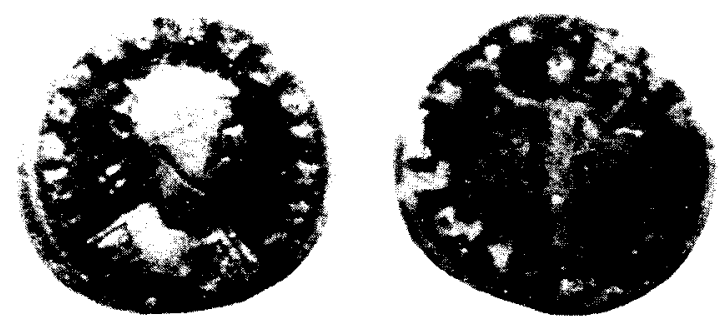

8
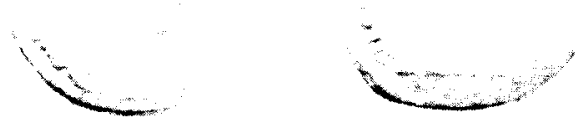

9
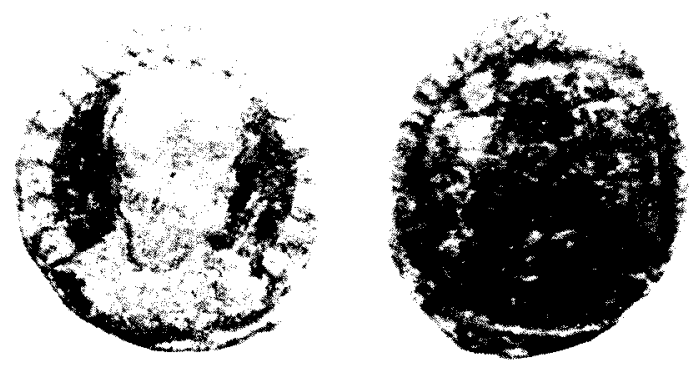

10 

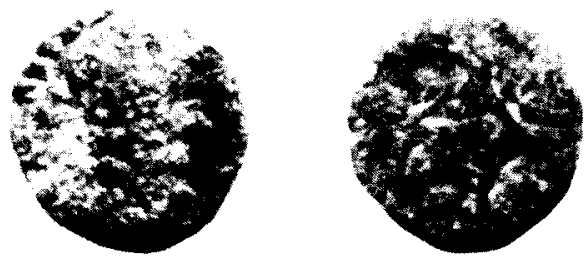

11
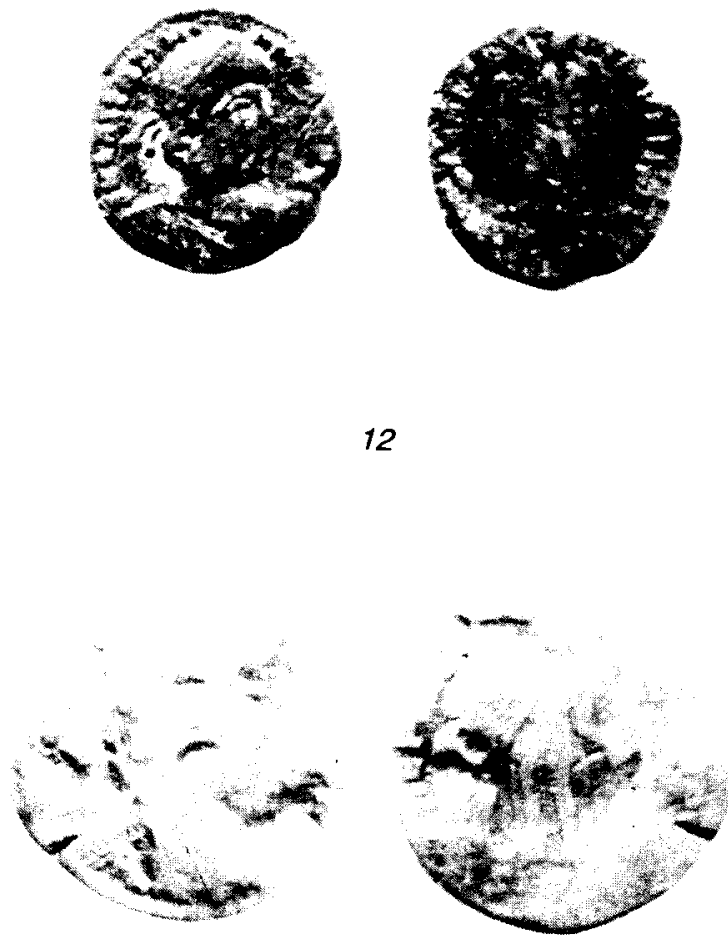

13 

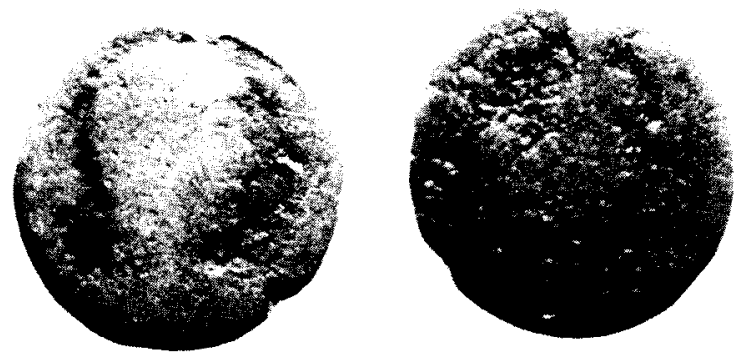

14
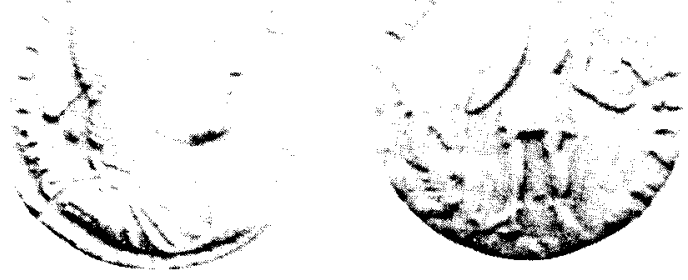

15
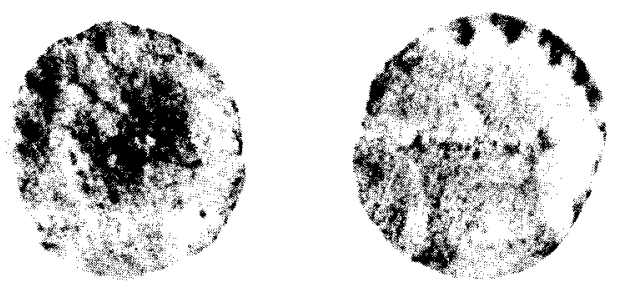
En todo el conjunto no se observa ninguna repetida. Esta circunstancia nos podria llevar a pensar que es un factor típico de una colección, pero debemos señalar que, cuando nos las propusieron para su estudio, estaban todavía sin clasificar, por tanto era raro que, sin conocer de que monedas se trataban, se hubiese procedido con anterioridad a una selección.

Las únicas novedades que cabria destacar es la presencia de un as de Galba y un sextercio de Maximino I, siendo las dos representaciones de estos emperadores las primeras que aparecen, hasta ahora, en toda la provincia.

Si agrupamos por siglos el total de las monedas encontradas en Segovia (fig. 1), vemos que, por su volumen, los siglos III y IV d.C. son los casi únicamente representados. A estos dos siglos correspondería el 81,81 por 100 del total y a cada uno de ellos el 40,90 por 100 , ya que el resto de los periodos, salvo el siglo I, sólo cuentan con un ejemplar.

La existencia de esta proporción es algo habitual ${ }^{12}$, puesto que el crecimiento de las acuñaciones durante el siglo III y más todavía durante el siglo iv es algo que está demostrado ${ }^{13}$.

Al presentarlas de una forma más pormenorizada, según los distintos períodos cronológicos (fig. 2), nos encontramos con que la etapa más

\begin{tabular}{|cccccc|}
\hline Siglos & Segovia & Segovia-Museo & N. ${ }^{\circ}$ total & $\%$ & Espirdo \\
\hline I a.C & 1 & 1 & 2 & $9,09 \%$ & \\
I a.C. & 1 & & 1 & $4,54 \%$ & \\
II & 1 & & 1 & $4,54 \%$ & \\
III & 5 & 4 & 9 & $40,90 \%$ & 1 \\
IV & 7 & $1+1$ (frustra) & 9 & $40,90 \%$ & \\
\hline Totales & 15 & 7 & 22 & & 1 \\
\hline
\end{tabular}

Fig. 1. Monedas romanas aparecidas en la ciudad de Segovia.

12 No entramos en otras circunstancias lógicas que se producen al emprender unas obras, dentro de la ciudad, bien se trate de zanjas o construcciones como es que afloren en las capas menos profundas los materiales arqueológico más próximos en el tiempo.

13 Ripolles AlegRe, P. P. La circulación monetaria en las tierras valencianas durante la antigüedad. Barcelona 1980, págs. 160-171; otro ejemplo lo tenemos en HIERNARD, J., "Conimbriga. Monedas y excavaciones antiguas (1930-1944 y 1959-1962) y franco-portuguesas (1964-1968)", Symposium Numismático de Barcelona (27 y 28 de febrero de 1979). Barcelona 1979, vol I, págs. 139-151. 


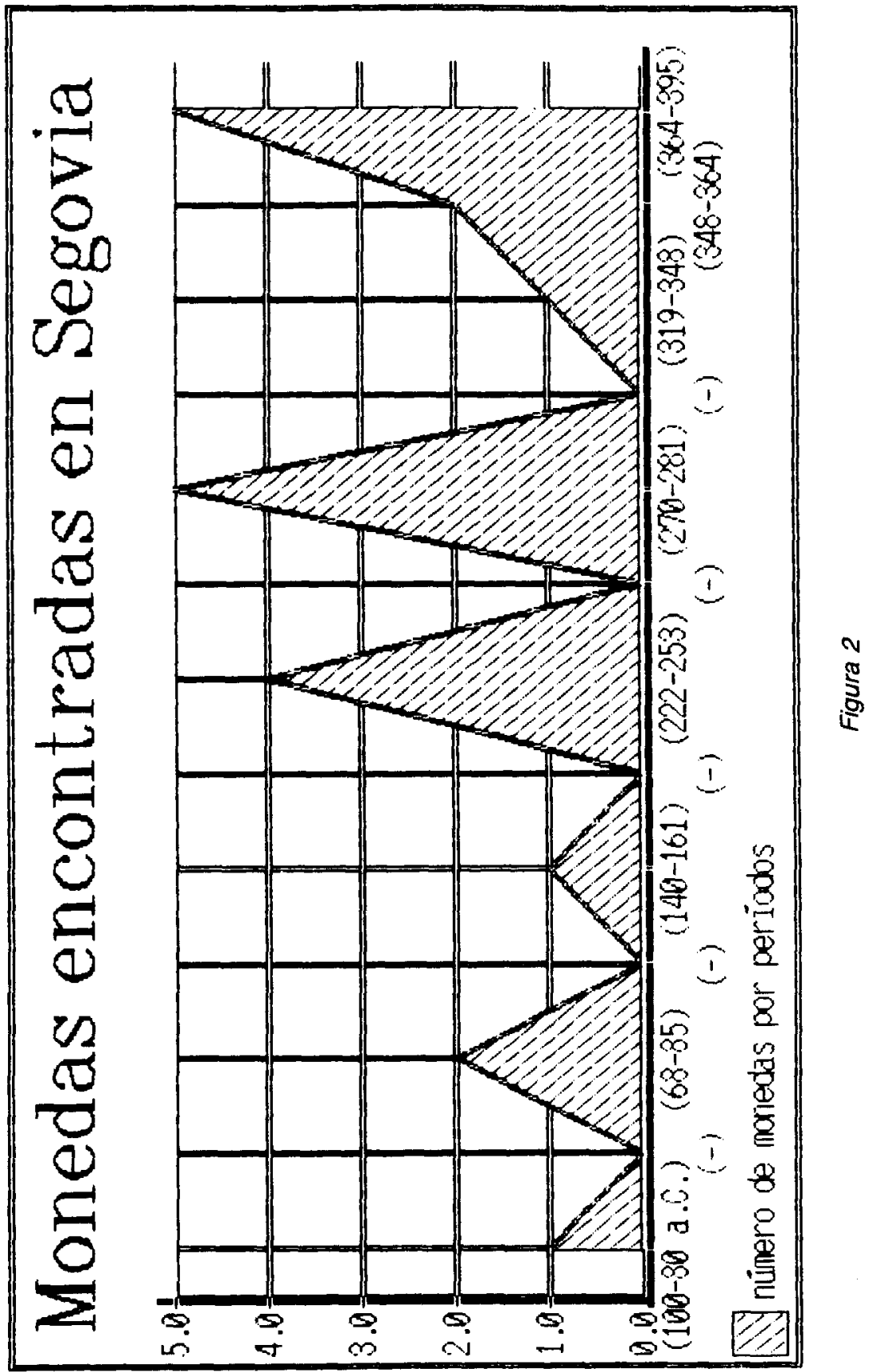


fértil es desde el año 222 al 281, correspondiendo a estos 59 años el 0,15 por 100 de monedas por año. La segunda será la comprendida entre los años 319 y 395, mucho más amplia, puesto que abarca 76 años con una media de 0,10 por 100 de monedas por año. Si observamos la curva que se produce al realizar un gráfico con las medias por año en cada uno de los períodos (fig. 3), vemos que destaca en gran medida el comprendido entre el 270-281.

Con estos datos da la impresión de que en Segovia existe una cierta permanencia de monedas en el período anteriormente señalado del siglo III (222-281), apareciendo en una proporción más alta en la capital que en el resto de la provincia ${ }^{14}$. Aunque creemos que esta conclusión puede que no sea definitiva por tratarse de una representación pequeña de piezas y que, además, no se han recogido en excavaciones sistemáticas. Sin embargo, esta misma tendencia aparece en otro núcleo urbano como Clunia $^{15}$. Si comparamos de forma generalizada estos hallazgos con los de Coca, que es el centro con mayor información de la provincia ${ }^{16}$, observamos que estos dos períodos aparecen como los dos más destacados, pero en proporción inversa, es decir, con marcada diferencia a favor del siglo iv. Esto mismo ocurre en Duratón ${ }^{17}$.

De momento, es un dato a tener en cuenta para posteriores estudios sobre circulación, en los que cabria plantearse el interrogante de si el creciente aumento monetario que se produce durante el siglo $\mathrm{IV}$ en las zonas rurales o villas romanas de esta provincia es sinónimo de auge económico, y si éste, como puede suceder en este caso, se produce en detrimento de algunas ciudades ${ }^{18}$.

14 Sagredo san Eustaduio, L. y Arribas Clemente, E.: Circulación y evolución monetaria..., obra citada, pág. 180.

15 Gurt Esparraguera, J. Ma. Clunia III. Hallazgos monetarios. La romanización de la Meseta Norte a traves de la circulación monetaria en la ciudad de Clunia. EAE 145, 1985, pág. 21.

${ }^{16}$ Blanco Garcia, J. F., Coca ..., obra citada, pág. 23, fig. 20: o la misma en: Moneda y circulacion..., obra citada, gráfico I. A estas habria que añadir las presentadas por $S_{A}$. GREDO, L. y ARRIBAS, E., Circulación.... obra citada, págs. 95-99; y las estudiadas por: SA GREDO, L. Y ZUMEL, L., "Estudio de monedas ...", obra citada, págs. 9-32.

17 Molinero Pérez, A., La necrópolis visigoda .... págs. 117-121 y 134-135 y nota 67 y Aportaciones de las excavaciones ..., EAE 72, Madrid 1971, págs. 25-49, 74, 77, 78 y 80. Todas ellas depositadas en el Museo Arqueológico de Segovia. Algunas de éstas se pueden estudiar mejor en: Sagredo san Eustaquio, L. y Arribas Clemente, E.: Circulación y evolución ..., págs. 100-137 y cuadro $n .^{\circ} \mathrm{VI}$ en la pág. 180.

18 Tenemos como ejemplo el trabajo realizado con los hallazgos monetarios de las excavaciones en la ciudad romana de Verulamium, (Hertforshire), KEAY, S.: "La circulación monetaria romana urbana y en el territorium: un estudio en contraposición", Symposium 
Estudio de algunos hallazgos numismáticos encontrados en Segovia

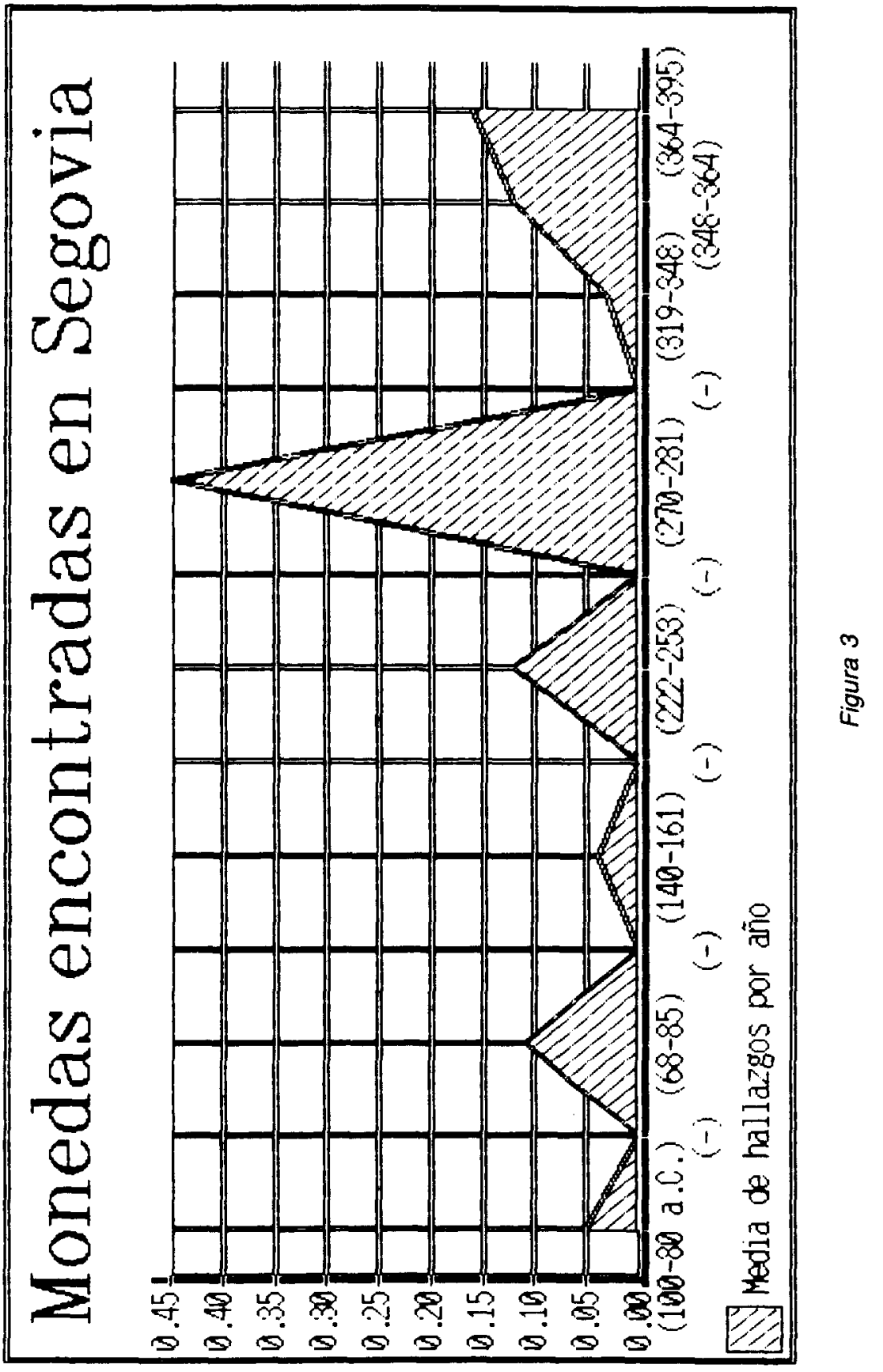


Presentando el total de las cecas conocidas en las monedas del siglo III y IV encontradas en Segovia tendríamos:

Siglo III

(Imitación)

Siglo IV

$\begin{array}{cccc}\text { Gala } & \text { Ticinum } & \text { Roma } & \text { Siscia } \\ 1 & 1 & 3 & 1 \\ 1 & & & \end{array}$

Tr. Lg. Ar. Galo-itálica Ss. C.
1

12

Siguiendo el cuadro comprobamos que las cecas italianas son las que predominan durante el siglo III, así como las galas están presentes en la primera parte del siglo Iv. Sin embargo, a finales de este siglo, desde el 378 al 387, son las cecas orientales las que nos abastecen, puesto que de las cuatro identificadas tres son de Siscia y Constantinopla y la cuarta de Arlés, acuñada por Magno Máximo. Esta tendencia observada aqui a favor de las cecas orientales ya fue confirmada, de manera general para toda la Hispania romana, en un trabajo anterior ${ }^{19}$.

Entre las leyendas aparecidas en los reversos la única que se repite es REPARATIO REIPVB, en tres piezas de finales del siglo IV.

En lo que respecta al metal, no se ha encontrado, hasta ahora, ninguna de oro ni plata, puesto que son todas de bronce o con distintas aleaciones sin precisar, como el antoniniano de Probo y las monedas de Claudio II.

\section{CONCLUSIONES}

Después de toda esta exposición, y siendo todo lo cautos que nos obliga la escasez y la poca claridad de las fuentes utilizadas, podriamos señalar que hay indicios para pensar que Segovia durante el siglo iv no sigue la misma marcha que Coca, situada más al Norte de la provincia, en su economía monetaria. Por otra parte, lo que ocurre en Coca estaría

Numismático de Barcelona (27 y 28 de febrero de 1979) vol. I, Barcelona 1979, págs. 239256, donde se contraponen a los resultados de otros hallazgos en villas romanas próximas.

19 Abad VARelA, M., Circulación monetaria en la Hispania romana del siglo IV d.C., Madrid 1989, págs. 1572 y 1573 , microficha n. ${ }^{\circ} 13$. 
en consonancia con lo mismo que pasa en Arcóbriga (Monreal de Ariza, Zaragoza), otra ciudad del interior, o en Conimbriga $u$ otras en torno al Mediterráneo, como es el caso de Tarragona y Pollentia ${ }^{20}$.

Sin embargo, Segovia, al igual que Clunia, estaría dentro de la línea de las ciudades que durante la segunda mitad del siglo iv decaen, a juzgar por la escasez del número de sus hallazgos que dan una media por año muy baja. Esto estaría en contraposición con la intensa producción de monedas llevadas a cabo durante el siglo iv en las cecas del Imperio ${ }^{21}$. Por otro lado, tendriamos que esta disminución del porcentaje de moneda por año que se da en Segovia durante el siglo IV, no se produce en las zonas rurales o villas romanas de la provincia, como es el caso de Duratón o Madrona ${ }^{22}$.

En cuanto a las cecas de las que tenemos constancia en estos hallazgos, no hay nada especial que reseñar, siguen en su abastecimiento la misma linea que se detecta de forma general en toda la Península.

Finalmente, con este pequeño trabajo pretendemos también llamar la atención para que todos los hallazgos numismáticos que se produzcan no queden relegados al olvido y se publiquen. Esto permitiría señalar el contexto estratigráfico donde se encontraron. Cumplido este objetivo, podremos disponer, a la larga, de fuentes más documentadas y nos será más fácil superar con acierto esta pequeña aproximación a la economía monetaria de Segovia que, al final, redundará en un mejor conocimiento de su historia.

20 Abad Varela, M., Circulación ..., obra citada, pág. 1570 y 1571 , microficha $n .^{\circ} 13$.

${ }^{21}$ Abad Varela, M., Circulación ..., obra citada pág. 1579 y 1580 , figuras n. 100 y 101

22 Sagredo san Eustaduio, L. y Arribas Clemente, E., Circulación y evolución ..., págs. 138-148 y pág. 180 , cuadro n. $^{\circ} \mathrm{Vl}$. 


\section{BIBLIOGRAFIA}

BAstien, P., 1983: Le monnayage de Magnence (350-353), Wetteren.

MatTINGLY, H., 1968: Coins of the Roman Empire in the Bristish Museum, vol. I, Augustus to Vitellius, Londres, reimpresión revisada de 1976, vol. IV, Antoninus Pius to Commodus, Londres.

BRuck, G., 1961: Die Spätrömische Kupferprägung, ein Bestimmungsbuch für schlecht erhaltene Münzen, Graz (Austria).

Hill, P. V., Kent, J. P. C. y CARson, R. A. G., 1978: Late Roman Bronze Coinage, Londres.

Sutherland, C. H. V., 1984: The Roman Imperial Coinage, vol. I, 31 BCAD 69, Revisado, Londres. Mattingly, H. y Sydenham, E. A., 1926: vol. II, Vespasian to Hadrian, Londres. vol. III, Antoninus Pius to Commodus, 1930; vol. IV, 2. ${ }^{\text {a }}$ parte, Macrinus to Pupienus, 1938; vol. IV, 3. ${ }^{2}$ parte, Gordian III-Uranius Antoninus, 1949; WEBB, P. H. 1933: vol. V, 2. ${ }^{a}$ parte, Probus-Amandus, Londres. BruUn, P. M., 1966: vol. VII, Constantine and Licinius, Londres.

Robertson, A. S., 1977 y 1978: Roman Imperial Coins in the Hunter Coin Cabinet, University of Glasgow, vol. III, Pertinasc to Aemilian, Glasgow; y IV, Valerian I to Allectus, Glasgow. 